\title{
Multiscale Modeling for Mechanical Properties of Cancellous Bone Based on the Schwarz Surface
}

\author{
ZhiQiang Huang ${ }^{1,2}$,Yufeng $\mathrm{Nie}^{1}$, Zihao Yang ${ }^{1}$, YiQiang Li $^{1}$ and NiuPing Guo ${ }^{3}$ \\ ${ }^{1}$ Department of Applied Mathematics, Northwestern Polytechnical University, Xi'an, 710072, China \\ ${ }^{2}$ Department of Mathematics, TaiYuan University of Science and Tcehnology, TaiYuan, 030024, China \\ ${ }^{3}$ School of Statistics, Xi'an University of Finances and Economics, Xi'an, 710100,China
}

\begin{abstract}
Microstructure and the mechanical properties of cancellous bone were modeled by multiscale finite element method in this paper. Microstructure of the cancellous bone determines its mechanical properties and a rational geometry modeling of the cancellous bone is fundamental to predict the material properties. In this paper, we generate a new parameterized microstructural unit cell model based on triple periodic minimal surfaces (TPMS) which is established by the Schwarz surface with various volume fractions. This model truly simulates the microstructure of the cancellous bone and satisfies some biological and mechanical requirements. By using the new unit cell model, the second-order two-scale (SOTS) method is applied to predict the mechanical properties of cancellous bone. The predictive the mechanical properties of numerical results are in good agreement with the experimental data reported in literature, which demonstrate that the established unit cell model is applicable and the second-order two-scale method is valid to predict the mechanical properties of cancellous bone.
\end{abstract}

\section{Introduction}

The investigation of the effective properties of cancellous bone is an important topic in biomechanics which has been especially intensive as the requirement for clinical practice in recent years, for example, it may lead to the early detecting of osteoporosis[1] and the artificial orthopaedic devices, especially the complex problem of bone implant interaction emerged and recalled the importance of the interrelations between biological and mechanical properties[2] .

To understand and study the mechanical and biological functions of cancellous bone, it is essential to clarify the relationships between the mechanical properties and the structural changes at the microstructural level. Such understanding is important to analyzing implants and manufacturing synthetic bone substitutes, at the same time it can serve engineers as a guide in designs of novel artificial biomimetic materials for a wide range of engineering applications [3]. For the investigation of cancellous bone properties different approaches are developed, one of them being of experimental methods, the other ones concentrating upon developing a convenient mathematical or mechanical model. In the aspect of numerical simulation, various analytical and computational models have been proposed to predict mechanical properties of bone at these different structural scales. Sandra Ilic(2010) considers the application of multiscale finite element method to the modeling of cancellous bone as an alternative for Biot's model[4]. Hamed,Elham(2010) predict analytically the effective elastic constants of cortical bone by modeling its elastic response at these different scales[3]. A. Jaziri (2012) propose a new elastoplastic damage coupled model for the modelling of trabecular bone behavior which the damage is carried out thanks to the limit analysis based on the MCK criterion[2]. Sansalone(2014) proposes a new methodology which coupling modeling and micromechanical homogenization to estimate the material properties of bone while taking into account the uncertainties on the bone micro- and nanostructure[5] .

Due to the complicated geometry structure of cancellous bone, different mode of the unit cell can be proposed for its modeling. Most of geometric models were proposed by describing the cancellous bone using the terms "rod-like" and "plate-like" for a subjective classification of cancellous bone. Mclehaeny(1970) described the unit cell as the cubic form which relationships was predicted between bone density and Young's modulus of cancellous bone[6]. Beaupre and Hayes (1985) think the unite cell as a cell body containing spherical cavity[7]. Sandra Ilic (2010)are described for the unit cell as a cubic form with the side length which treat the solid frame as a system of thin walls whose thickness and width can be varied.[4]The other unit cell was used frequently to include Bar-Net model, Plate-Frame-Hole model, Honeybomb model, Plate-bar model etc.

However, these techniques are based on the assumption that all trabecular in the different position bones are either in the form of plates or rods, the 
microstructure in above models is very simplified and thus less precise results are obtained. An ideal unit cell model structure of cancellous bone should have appropriate porosity and pore-structure, to this purpose, a periodic embedded Schwarz surface (see the Fig.2)was established as the unit cell in our model, because of the Schwarz surface is more permeable, it also has a smooth surface to open the portion of the tissue which would facilitate the inflow of nutrients and the disposal of metabolic waste [8]. Most important of all, the new unit cell meet satisfy some biological and mechanical requirements of the cancellous bone.For the past few years, the second-order two-scale (SOTS) analysis method is introduced [Cui, Shin and Wang (1999)] to predict the physical and mechanical properties of periodic composites[8]. Microstructural modeling and mechanical properties computation of three-dimensional 4-directional braided composites were studied[9]. By the second-order correctors, the microscopic fluctuation of physical and mechanical behaviors inside the materials can be captured more accurately. In this paper, a new parameterized microstructural unit cell model is established, Schwarz primitive surface and it truly simulates the microstructure of cancellous bone. The parameterized design for the unit cell model is implemented by employing the TETGEN software. And then based on the established model and the mechanical parameters are predicted by using the second-order two scale method.

\section{Microstructure analysis of cancellous bone}

Structurally, cancellous bone is considered like a composite material with a complex structure. Form the mesoscale, ranging from hundred micrometres to several millimetres, or larger, depending on the bone size, consists of a porous network of trabeculae (Fig.1.). The pores, typically in the order of $1 \mathrm{~mm}$, are filled with bone marrow, fat and bone cells. In cortical bone, this level represents randomly arranged osteons embedded in an interstitial lamella, with some resorption cavities, all surrounded by a circumferential bone.
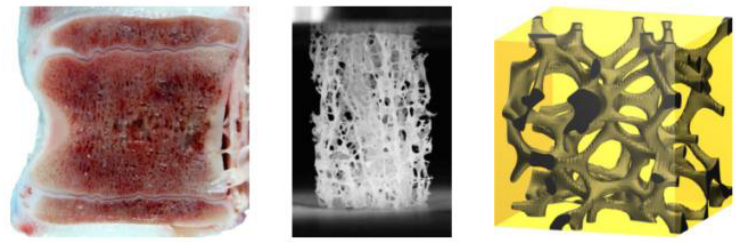

Figure 1. Specimens of trabecular bone obtained from vertebrae[10]

\section{Unit cell modeling of cancellous bone}

Hyperbolic surfaces have attracted the attention of physicists, chemists and biologists as they commonly exist in natural structures. Amongst various hyperbolic surfaces, minimal surfaces are the most studied, these surfaces are known as Triply Periodic Minimal Surfaces (TPMS). As the simulation of cancellous bone by the multiscale FE, it is important for unit cell of modelling the cancellous bone to have the suitable pore size, sufficient porosity, and high pore interconnectivity. The triply periodic minimal surface have been received much attentions in the recent biomaterial literatures and provide good analytic description of highly porous structures[11], however, there were few reports about the research on property of the cancellous bone simulation used these TPMS.

In a general way, a periodic surface can be generally defined as

$$
\phi(\mathbf{r})=\sum_{k=1}^{K} \mathbf{A}_{k} \cos \left[2 \pi\left(\mathbf{h}_{k} \cdot \mathbf{r}\right) / \lambda_{k}+p_{k}\right]=\mathrm{C}
$$

An important sub-class of TPMS is those that partition space into two disjoint but intertwining regions that are bi-continuous. An example of such surfaces includes the so-called Schwartz G surface defined as

$$
\begin{aligned}
& \phi(\mathrm{x}, \mathrm{y}, \mathrm{z})=\sin 2 \pi \mathrm{x} \cos 2 \pi y+\sin 2 \pi y \cos 2 \pi z \\
& +\sin 2 \pi z \cos 2 \pi x=C
\end{aligned}
$$

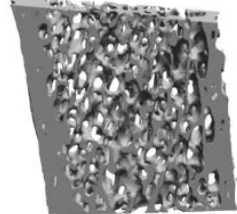

(a)

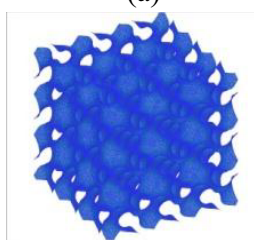

(d)

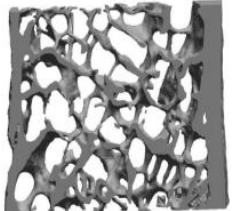

(b)

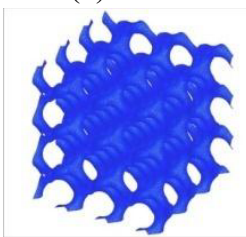

(e)

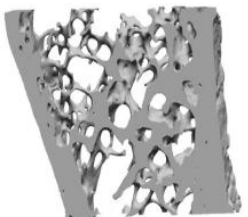

(c)

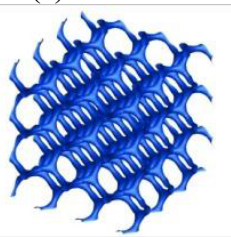

(f)
Figure 2. MicroCT imaging of iliac bone (a).In a young and healthy subject: the cortices are thick and the trabecular network is dense, (b).In a postmenopausal osteoporosis, the holes inside the network (c).In a male with idiopathic osteoporosis, note the conversion of plate into rods, although the connectivity is rather well even if trabecular are thin.[12] (d-f).The microstructure of the unit cell based on the Schwarz surface of a different porosity was used in this work.

There are the potential benefits of Schwarz surface based pore architectures which were put forward to taking advantage of these newly proposed pore geometries as unit cell to simulate the cancellous bone. Viewed from biology and biomechanics, beneficial properties can be provided by TPMS for the cancellous bone as attractive candidates for biomorphic porous architectures.

The advantages of the method utilized in the after section are satisfy some biological and mechanical requirements, such as: From the viewpoint of morphology, the unit cell based on Schwarz surface is very vividly to simulating the cancellous bone structure, and that it is important for unit cell of modeling the cancellous bone to have the suitable pore size, sufficient porosity, and high pore interconnectivity. From the 
viewpoint of mechanical requirements, the researched results imply that the Schwarz surface more stable structures [8] which the unit cell should consider the sufficient mechanical strength and stiffness for the cancellous bone to support the growing tissue. From the viewpoint of biological requirements, the permeability of the unit cell should be high enough to provide superior diffusion which would facilitate the inflow of nutrients and the disposal of metabolic waste. The surface of the unit cell based on Schwarz surface was sufficiently smooth which would be very beneficial to inflow and outflow of nutrients and metabolic waste. Micro-structure with a high porosity of the unit cell provides more space for cells to move into and begin to thrive, the unit cell based on Schwarz surface has a large surface area which enables much more cell attachment and growth and subsequently sufficient for tissue repair. From the viewpoint of self-optimizing of bone tissue, above these characters of the unit cell based on Schwarz surface consistent with demand of modeling cancellous bone which must satisfy some biological and mechanical requirements, at same time, which provide insights into the reason behind the natural choice by biological systems.

\section{Multiscale modeling and FE analysis}

\subsection{Second-order two-scale formulation}

From solid mechanics, the elasticity problem of the structures made from periodic composites can be expressed as follows

$$
\begin{cases}\frac{\partial}{\partial x_{j}}\left(C_{i j h k}^{\varepsilon}(x) \frac{1}{2}\left(\frac{\partial u_{h}^{\varepsilon}(x)}{\partial x_{k}}+\frac{\partial u_{k}^{\varepsilon}(x)}{\partial x_{h}}\right)\right)=f_{i}(x) & x \in \Omega \\ \mathbf{u}^{\varepsilon}(x)=\overline{\mathbf{u}}(x) & x \in \Gamma_{1} \\ \sigma(u) \equiv v_{j} C_{i j h k}^{\varepsilon}(x) \frac{1}{2}\left(\frac{\partial u_{h}^{\varepsilon}(x)}{\partial x_{k}}+\frac{\partial u_{k}^{\varepsilon}(x)}{\partial x_{h}}\right)=p_{i}(x) & x \in \Gamma_{2} \\ \Gamma_{1} \cap \Gamma_{2}=\varnothing, \quad \Gamma_{1} \cup \Gamma_{2}=\partial \Omega & \end{cases}
$$

where $i, j, k, l=1,2,3 ; \Omega$ is composed of entire $\varepsilon$-size cells, $\mathbf{u}^{\varepsilon}(x)$ is the displacement solution; $f_{i}(x)$ is a body force independent of $\varepsilon ; C_{i j h k}^{\varepsilon}(x)$ are the elastic coefficients; $\Gamma_{1}$ and $\Gamma_{2}$ denote boundaries of $\Omega$ where displacement $\overline{\mathbf{u}}(x)$ and loads $p_{i}(x)$ are prescribed, respectively; $v_{j}$ are the normal direction cosines of $\Gamma_{2}$.

Let $y=x / \varepsilon \in Y$ denotes the local coordinates on the unit cell. Then $\mathbf{u}^{\varepsilon}(x)=\mathbf{u}(x, y)$ and $C_{i j h k}^{\varepsilon}(x)=C_{i j h k}(y)$. the secondorder two-scale approximate solution can be expressed as

$$
\mathbf{u}^{\varepsilon}(x)=\mathbf{u}_{0}(x)+\sum_{l=1}^{2} \varepsilon^{l} \sum_{\alpha_{1} \cdots \alpha_{l}=1,2,3} \mathbf{N}_{\alpha_{1} \cdots \alpha_{l}}(y) \frac{\partial^{l} \mathbf{u}_{0}(x)}{\partial x_{\alpha_{1}} \cdots \partial x_{\alpha_{l}}}
$$

Where $\mathbf{u}_{0}(x)$ is the homogenized solution that was defined on $\Omega, \mathbf{N}_{\alpha_{1}}(y)$ and $\mathbf{N}_{\alpha_{1} \alpha_{2}}(y)$ are matrix-valued functions defined on the unit cell $Y$. And $\mathbf{N}_{\alpha_{1}}(y)$, $\mathbf{N}_{\alpha_{1} \alpha_{2}}(y)$ and $\mathbf{u}_{0}(x)$ are determined in the following ways

For $l=1, \mathbf{N}_{\alpha_{1} m}(y)\left(\alpha_{1}, m=1,2,3\right)$ are solutions of the following problem

$$
\left\{\begin{array}{l}
-\frac{\partial}{\partial y_{j}}\left[C_{i j h k}(y) \frac{1}{2}\left(\frac{\partial \mathbf{N}_{\alpha_{1} k m}(y)}{\partial y_{h}}+\frac{\partial \mathbf{N}_{\alpha_{1} h m}(y)}{\partial y_{k}}\right)\right] \\
=\frac{\partial}{\partial y_{j}}\left(C_{i j m \alpha_{1}}(y)\right), \quad y \in Y \\
\mathbf{N}_{\alpha_{1} m}(y)=0, \quad y \in \partial Y
\end{array}\right.
$$

From $\mathbf{N}_{\alpha_{1} m}(y)\left(\alpha_{1}, m=1,2,3\right) \quad, \quad$ the homogenized parameters $\hat{C}_{i j h k}$ are calculated by the following formula

$$
\hat{C}_{i j h k}=\frac{1}{|Y|} \int_{Y}\left(C_{i j h k}(y)+C_{i j m \alpha_{1}}(y) \frac{1}{2}\left(\frac{\partial \mathbf{N}_{h m k}(y)}{\partial y_{\alpha_{1}}}+\frac{\partial \mathbf{N}_{h \alpha_{1} k}(y)}{\partial y_{m}}\right)\right) d y
$$

\subsection{Finite element meshes of cancellous bone}

Due to the complexity of the geometry model of the cancellous bone were developed, the efficiency and precision of SOTS calculation was importantly influenced by the quality to generate meshes. In this paper, the TETGEN software package was used to generate the FE meshes of the model. The geometry model of the unit cell developed by using the MATLAB software was imported into TETGEN software, and then the model with the mineralized collagen fibrils volume fraction $21 \%$ was meshed by 112413 tetrahedron elements shown in Fig. 3.

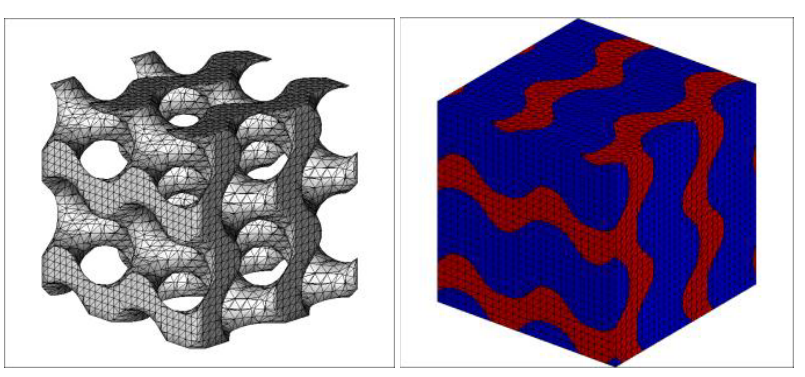

Figure 3. FE meshes of the unit cell of cancellous bone. The meshes of (a) mineralized collagen fibrils and (b) the entire unit cell

\section{Numerical examples and performance analysis}

In this section, the algorithm procedure of the SOTS method for predicting the mechanical properties of cancellous bone is presented, and then some numerical example are given. 


\subsection{Numerical examples}

The finite element method was used to evaluate and simulate the mechanical parameters under different conditions such as volume fractions. We present a multiscale modeling approach for cancellous bone with an appropriate porosity and mechanical properties made from a two-phase composite of hydroxyapatite (HAp) embedded in a collagen matrix. We assume an isotropic linear elastic behavior for both collagen and hydroxyapatite, the material properties used in this paper of the collagen and the hydroxyapatite are summarized in Table 1.

Table 1. Properties of bone components selected in this work[13]

\begin{tabular}{|c|c|c|l|}
\hline Material & $\begin{array}{l}\text { Elastic } \\
\text { modulus } \\
(\mathrm{GPa})\end{array}$ & $\begin{array}{c}\text { Poission's } \\
\text { ratio }\end{array}$ & $\begin{array}{l}\text { Volume } \\
\text { fraction (\%) }\end{array}$ \\
\hline Collagen & 1.5 & 0.28 & $90 \sim 40$ \\
\hline $\begin{array}{c}\text { Hydroxyapatite } \\
\text { (HAp) }\end{array}$ & 114 & 0.23 & $10 \sim 60$ \\
\hline
\end{tabular}

\subsection{Effective material parameters}

In Fig.4, the results corresponding to the unit cell with constants $C$ in the interval $0.6-0.92$ are considered. Dependence of Young's modulus and shear modulus on porosity is a smooth, monotonically decreasing function, while Poisson's ratio shows opposite behavior and it increases with increasing porosity.Young's modulus takes the values in the interval 753.21-3029.83 N/ $\mathrm{mm}^{2}$, the shear modulus 384.38-1381.32 N/mm ${ }^{2}$ and Poisson's ratio $0.209-0.252$ for the porosity from 0.4667 0.8156 . These results agree well with the results obtained by Ashman and Rho (1988), that the structural elasticity modulus of cancellous bone has the values in the interval $985-2,110 \mathrm{~N} / \mathrm{mm}^{2}$.

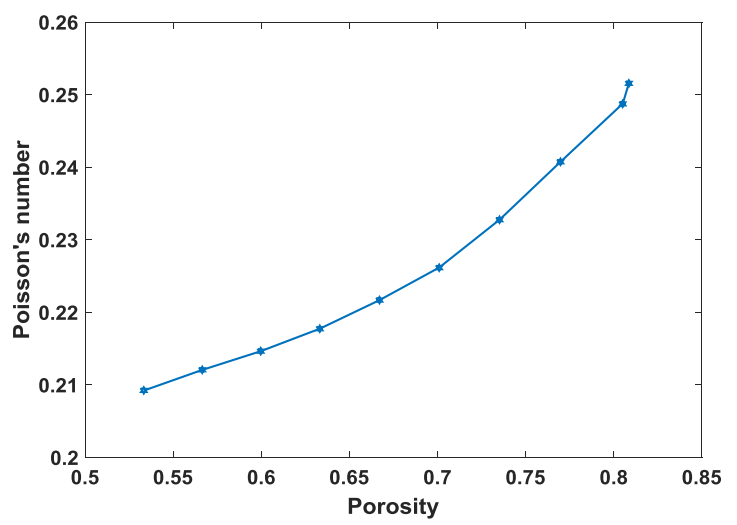

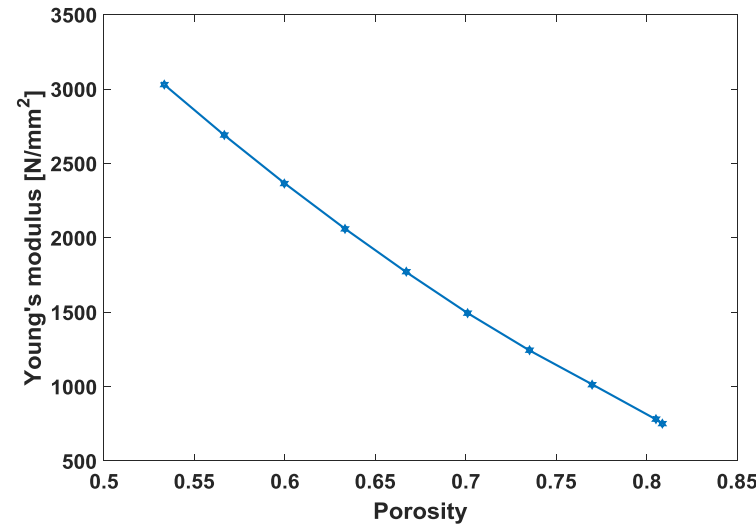

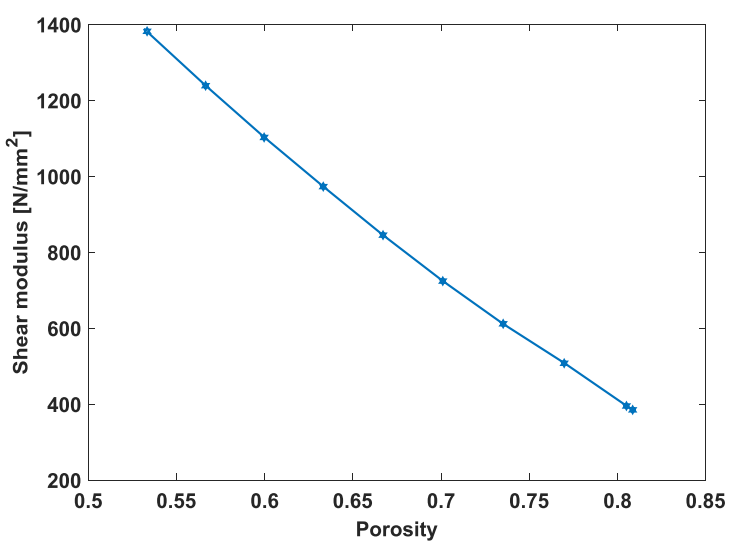

Figure 4. Change of effcetive material parameters versus porosity for the biphasic material

\section{Conclusions}

This study focuses on the determination of the relationship between mechanical functions and structure of bone tissues, it provides a significant improvement of the tools available for studying and understanding the mechanical functions of cancellous bone. In this paper, a new parameterized microstructural unit cell model is established and the SOTS method is applied to predict the mechanical properties of the cancellous bone. The geometry model of the unit cell developed by using the MATLAB software was imported into TETGEN software. And this model truly simulates the microstructure of the cancellous bone. From a modelling point of view, the advantages of the novel unit cell are satisfy some biological and mechanical requirements and the natural choice by biological systems. Based on these unit cell models, Poission's ratio and elastic modulus of cancellous bone are predicted by the SOTS method. The good agreement between the calculated results and experimental data and other research results, Moreover, the relations between different volume fractions and elastic constants are investigated. The results show that mechanical properties and volume fractions of cancellous bone components play an important role in its overall behavior. 


\section{Acknowledgement}

This work is supported by the National Natural Science Foundation of China (Grant No.11471262), and also supported by Center for high performance computing of Northwestern Polytechnical University.

\section{References}

1. Basaruddin, K.S., N. Takano, and T. Nakano, Stochastic multi-scale prediction on the apparent elastic moduli of trabecular bone considering uncertainties of biological apatite (BAp) crystallite orientation and image-based modelling. Comput Methods Biomech Biomed Engin. 18,2(2015).

2. A. Jaziri a , J.R.b., Multi-scale modelling of the trabecular bone elastoplastic behaviour under compression loading. European Journal of Computational Mechanics,21,3-6 (2012).

3. Hamed, E., Y. Lee, and I. Jasiuk, Multiscale modeling of elastic properties of cortical bone. Acta Mechanica, 213,1-2(2010).

4. Ilic, S.H., Klaus Gilbert, Robert, Application of the multiscale FEM to the modeling of cancellous bone. Biomech Model Mechanobiol. 9,1(2009).

5. Sansalone, V., S. Naili, and C. Desceliers, A stochastic homogenization approach to estimate bone elastic properties. Comptes Rendus Mécanique. 342,5(2014).

6. Shin,J., et al., Finite Element Analysis of Schwarz P Surface Pore Geometries for Tissue-Engineered Scaffolds. Mathematical Problems in Engineering. 2012,1-13(2012).

7. Hayes, G.S.B.W.C., Finite Element Analysis of a Three-Dimensional Open-Celled Model for Trabecular Bone. Journal of Biomechanical Engineering, 107,3 (1985).

8. Cui, J. Z.; Shin, T. M.; Wang, Y. L. Two-scale analysis method for bodies with small periodic configuration. Structural Engineering and Mechanics, 7, 6(1999)

9. Zihao Yang, J.C., Yufeng Nie, Microstructural Modeling and Second-Order Two-Scale Computation for Mechanical Properties of 3D 4-Directional Braided Composites.CMC,,38, 3(2013)

10. Schwen, L.O.,Multiscale elasticity modeling of trabecular bone. 2013.

11. Yoo, D., New paradigms in hierarchical porous scaffold design for tissue engineering. Mater Sci Eng C Mater Biol Appl, 33,3(2013).

12. Chappard,D.,etal.,Trabecular bone microarchitecture: a review. Morphologie. 92,299 (2008)

13. Hamed,E.,et al., Multi-scale modelling of elastic moduli of trabecular bone. J R Soc Interfece,9,72(2012). 